\title{
Mumps outbreak in Copperbelt province, Zambia: Epidemiological characteristics
}

\author{
Rufaro M. Chirambo ${ }^{a}$, Peter Songolo ${ }^{a}$, Freddie Masaninga ${ }^{a}$, Lawrence N. Kazembe ${ }^{\text {,** }}$ \\ ${ }^{\text {a }}$ WHO Country Office, Lusaka, Zambia \\ ${ }^{\mathrm{b}}$ Department of Statistics, University of Namibia, Windhoek, Namibia
}

\section{A R T I C L E I N F O}

\section{Keywords:}

Mumps outbreak

Risk factors

Immunogenicity

Copperbelt province

Zambia

\begin{abstract}
A B S T R A C T
Background: Mumps are known to occur in Zambia, but outbreaks are rare. Here we report on a recent mumps outbreak, between January and September, 2015.

Objective: The study was to verify the existence and extent of mumps outbreak in Copperbelt province; to describe the epidemiological characteristics of the outbreak; to identify risk factors associated with the illness; to confirm the etiology of the outbreak through appropriate laboratory testing; to inform disease control strategy and advocate for mumps, measles and rubella vaccine in Zambia, which has not yet been implemented.

Study design: A case report. Clinical records of cases suspected of mumps in the district were reviewed. Cases covered the first 36 weeks of 2015 (January to September, 2015). Charts were generated to capture the epidemic curve. Detailed analysis using data from Luanshya was carried, by calculating relative risk for the demographic factors and place of residence.

Results: Between week 1 and 36 of 2015, a total of 1651 cases of mumps were reported. The primary case was a four year old male child, and was diagnosed in the first week of January. Week 14 and 19 reported the highest number of cases, but the cases plateaued in week 33. Laboratory analysis provided a test positivity rate of $70 \%$. Most cases (71\%) were children aged between 1 and 9 years of age. This group was at least 3 times higher at risk than those $<20$ years.

Conclusion: Several factors may have contributed to the emergence of mumps outbreaks including intense exposures, overwhelming protection offered by the vaccine, combined with non-optimal vaccine coverage, low immunogenicity and waning immunity. It is therefore recommended that, first, continued surveillance be maintained so that cases are identified, contacts are traced, and inform implementation of appropriate infection control measures. Second, continued Information, Education and Communication (IEC) on good hygiene practices, modes of transmission and risk factors in schools and other public places is recommended. Third, there is need to establish local emergency health services linking schools and health facilities. Furthermore, health authorities should make concerted efforts to enable diagnostic tests for mumps within the country, while at the same time we advocate for Measles, Mumps \& Rubella (MMR) vaccine in Zambia.
\end{abstract}

\section{Background}

Mumps continue to be an important childhood disease in many parts of the world, mostly concentrated in children aged 5-9 years old. ${ }^{1-5}$ Adults can also be infected, but the prognosis is often severe. ${ }^{1-5}$ The disease is highly contagious and is characterized by fever and inflammation of the salivary glands. In some instances it could cause subclinical infections, and may lead to meningo-encephalitis, deafness and orchitis.

The incidence of mumps is rare, but outbreaks are common worldwide. In Zambia, no such outbreaks have been recorded since
1999. In this short communication, we describe the epidemiology of suspected mumps on the Copperbelt province, Zambia, from week 1-36 of 2015. Focus is also made on Luanshya district where most cases have been reported. The increased reports of mumps necessitated this clinical review. The analysis may inform disease control strategy and advocate for mumps, measles and rubella vaccine in Zambia, which has not yet been implemented.

\section{Objectives of the study}

The objectives of the study were to verify the existence and extent of

\footnotetext{
* Corresponding author.

E-mail address: lkazembe@yahoo.com (L.N. Kazembe).
} 


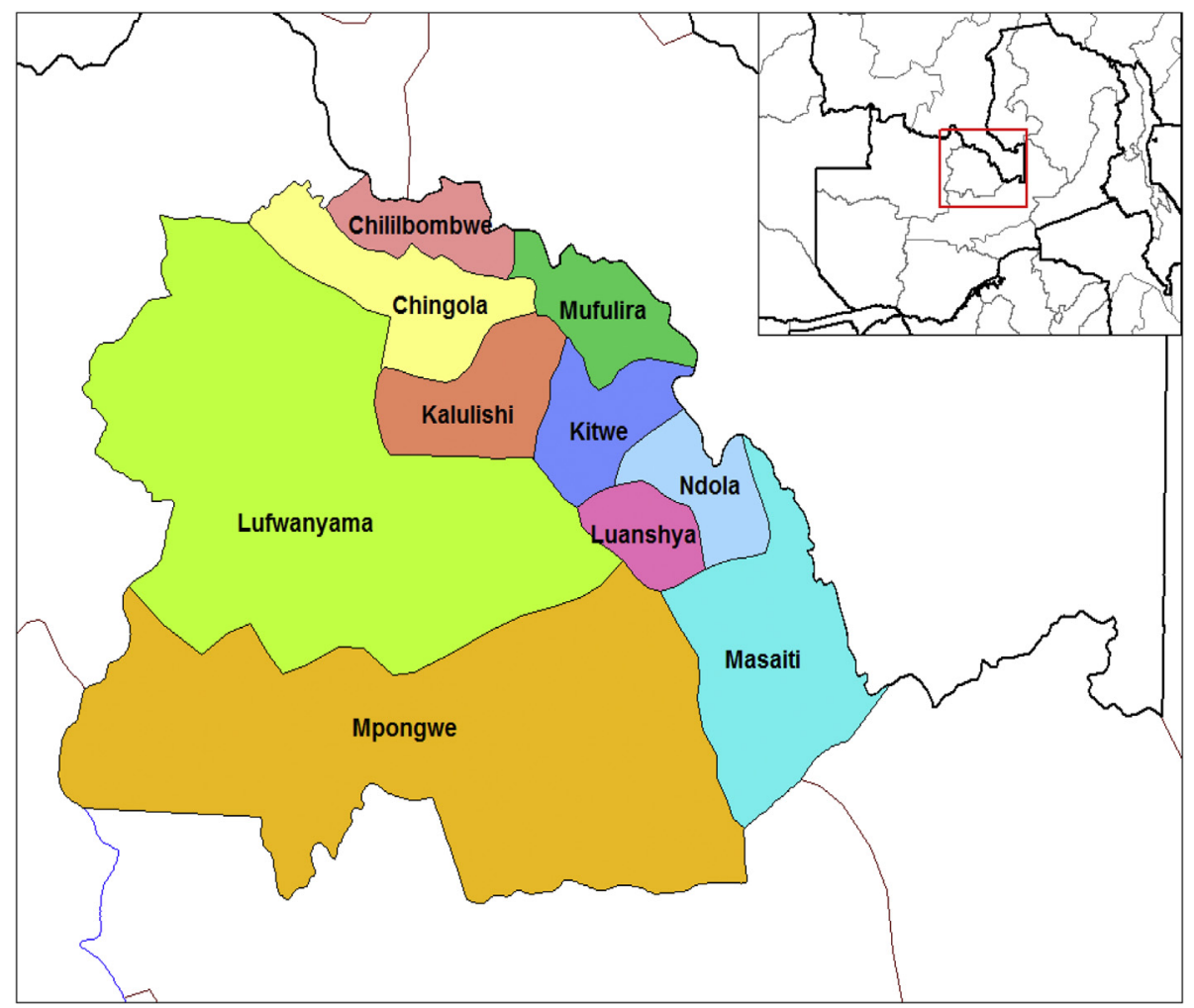

Fig. 1. Map of Copperbelt province and the corresponding districts where mumps cases were reported.

the outbreak; to describe the epidemiological characteristics of the outbreak; to identify risk factors that may be associated with the illness; to confirm the etiology of the outbreak through appropriate laboratory testing.

\section{Study design}

A case report of suspected mumps outbreak in the Copperbelt province, Zambia.

\subsection{Outbreak settings}

The Copperbelt province is situated in the northern - central Zambia, which is on the corridor linking with Lubumbashi in Democratic Republic of Congo (Fig. 1). The province has 10 districts. One of the districts which was most affected by mumps was Luanshya. It shares boundaries with Ndola and Kitwe to the north, Lufwanyama district to the west, Mpongwe to the south and Masaiti to the south-west as shown in the map (Fig. 1). It is $35 \mathrm{~km}$ away from the provincial capital Ndola, and $337 \mathrm{~km}$ from Lusaka, the capital city. The district has a population of 171,000 , with 32,300 households, and has one of the lowest annual percentage population growth $(0.3 \%)$ in Zambia. The population density is 188.8 people per square kilometres.

\subsection{Epidemiological investigation}

In April, 2015 an increase in reported suspected mumps cases was observed. Over 38 new cases were reported in week 16 (19th -26 th April, 2015). On 7th May, 2015, the Provincial Medical Officer and Ministry of Health were informed. On the 11th of May, 2015, a team from Tropical Disease Research Centre (TDRC) went to Luanshya District Health Office to investigate the outbreak: The team comprised of 2 Epidemiologists, 1 Field Epidemiology Training Program (FETP) resident, Surveillance Focal point person in Luanshya and an Environmental Health Technician (EHT).
The methods used to collect data were: (i) reviewed IDSR data and medical records (cases presented before 12th May, 2015); (ii) conducted interviews to collected data (cases presented after 11th May, 2015); (iii) collected specimen for laboratory confirmation from active patients between 10th to 23rd May, 2015.

\subsection{Case definitions}

Based on the World Health Organization definition, a case of mumps is an illness of unilateral or bilateral tender, self-limited swelling of the parotid or other salivary gland lasting two days or more, without other apparent causes. $^{1-3}$

\subsection{Risk factors for mumps}

We evaluated potential risk factors for mumps, including age at the start of the outbreak, gender, reporting health centre or clinic and residence. Other information, such as socio-economic condition and hygiene, immunization history, migrant, school contacts, among others, which could have permitted better insight on the epidemiological profile were inadvertently not collected at the time of examining cases. Our analysis included all cases that occurred in Luanshya district between January and June 2015.

\subsection{Laboratory investigations}

To verify the outbreak, a laboratory investigation was conducted. Seven blood samples were collected from active patients with clinical features compatible with mumps, shipped and analyzed by CDC in Atlanta. RT-PCR for mumps viral RNA test was conducted. Results came out on 10th July, 2015.

\subsection{Statistical analysis}

Descriptive analysis, using charts and tables were carried out. A chi- 
square test of association was conducted, with significant association evaluated at $\mathrm{p}<0.05$. We then carried out a Poisson regression to estimate the relative risk associated with each risk factor. Absence of overdispersion in the data was assessed using Dean's test. All statistical analyses were performed in R software. ${ }^{6}$

\subsection{Ethical clearance}

The study involved use of secondary data from the Integrated Disease Surveillance Reporting (IDSR) systems, and approval to use the data was granted by Ministry of Health in Zambia.

\section{Results}

\subsection{Index cases}

Five out of seven samples were positive, giving a test positivity rate of $70 \%$ (95\% CI: $45 \%-82 \%$ ). Mumps virus genotype D was confirmed in the case patients by presence of IgM (2), mumps RNA (1), or both (2).

The primary case was a male, aged 4 years, with an onset of symptoms reported on 1st January, 2015. The initial peak was on 5th January. Besides the January 5th peak, multiple peaks with an average of 12 cases were identified. The number of index cases reported in Luanshya in April ( $n=71,26 \%$ of total) exceeded the number reported in January $(n=54)$, February $(n=33)$, May $(n=54)$ and June $(n=18)$.

\subsection{Outbreak}

The overall mean rate of mumps per week was 45.8 cases $(95 \% \mathrm{CI}$ : $32.5-59.1$ cases). A total of one thousand, six hundred and fifty one $(1,651)$ cases of mumps were reported from Chililabombwe, Kalulushi, Luanshya, Mpongwe and Ndola districts of Copperbelt province, since January 2015, with Luanshya recording the highest number of cases (Fig. 2). The highest number of cases was reported in Weeks 14 $(n=99)$ and $19(n=99)$ as shown in the Epi curve (Fig. 2). Evidently, this was above the average weekly rate, and was sustained from week 14 to week 19. Other peaks, defined as values above the upper confidence limit, occurred in weeks of 27, 30, and 32. Fig. 3 shows that the estimated risk of an outbreak, remained low from week 1 to week 11, although the risk kept on increasing from the onset of the year. From week 12 , the risk was above zero $(R R=1)$ depicting a varying pattern to the end of the surveillance period (Week 36: September 2-September 8). There was, however, a sharp decline in weeks 20 and 21, reporting 34 and 35 cases respectively, compared to the number of cases in week 19 , about $65 \%$ reduction in the number of reported cases. The number of cases plateaued as from week 33, although these were above overall mean rate. A report from Luanshya which had the highest number of cases indicated that there were 18 cases reported in week 22, compared to 35 cases in week 21. Fig. 4 presents a snapshot of reported cases in week 36 in all districts of the Copperbelt province. Even after the cases reported have subsided, Luanshya (with 14 cases, $30.4 \%$ of cases in week 36 ) and Ndola (with 16 cases, $34.8 \%$ of cases in week 36 ) remained the top districts reporting suspected mumps. No death has been recorded so far. Clinical characteristics included buccal tenderness (41\%) and fever $>37.5^{\circ} \mathrm{C}(32 \%)$.

\subsection{Risk factors of mumps in Luanshya district}

Luanshya district experienced the highest number in the outbreak of mumps which began in January through May, 2015, with clustering of $35 \%$. Table 1 presents an epidemiological profile of cases identified in the district between January and June, 2015. The number of males and females infected were almost equal (51\% female and 49\% male). Most cases were reported in the age group 1-4 year olds (38\%) and 5-9 year olds (33\%). This outbreak was also characterized with cases arising among adolescents (6\%) and adults (11\%). The most affected residences were Mikomfwa and Roan Township, collectively reporting a cluster of $78 \%$ of all cases. The case distribution was similar at facility level, with most cases recorded at Mikomfwa clinic (44\%), and followed by Roan Section 9 health facility (33\%). There was zero-reported cases of complications resulting from the outbreak (Table 1).

Table 2 gives the relative risk of factors associated with mumps in Luanshya district. Please note that the variable sex was still included in the model, as a control variable, despite not being significant in the chisquare test. As expected, the risk for mumps was lower in males, but not significant $(R R=0.94,95 \%$ CI: 0.73-1.25). Children aged $1-4$ years and 5-9 years were at least 3 times more at risk of mumps that adults above the age of 20 years $(\mathrm{RR}=3.94,95 \% \mathrm{CI}: 2.34-6.23$ and $\mathrm{RR}=3.12$, 95\% CI: 2.11-4.93 respectively). Those who resided in Mikomfwa were more likely to have mumps than those in Mpatamato $(\mathrm{RR}=9.27,95 \% \mathrm{CI}: 4.85-15.82)$. The same pattern of association was observed for those living in Roan township and Kawama compared to those in Mpatamato (Table 2). With regards to the reporting clinic, all other clinics compared to Mikomfwa, were less likely to report cases of mumps (Table 2).

\section{Discussion}

In this study, we reported on mumps outbreak in the Copperbelt province, Zambia. The number of mumps cases increased from week 1 to week 36 of 2015 , with a plateau reached in week 33. The proportion of cases in Luanshya district was over one-third of all cases in Copperbelt province. The highest number of cases was reported in week 14 and 19 each with 99 cases from the entire province. The epidemic was confirmed with a test positivity rate of $70 \%(5 / 7$ tested). The

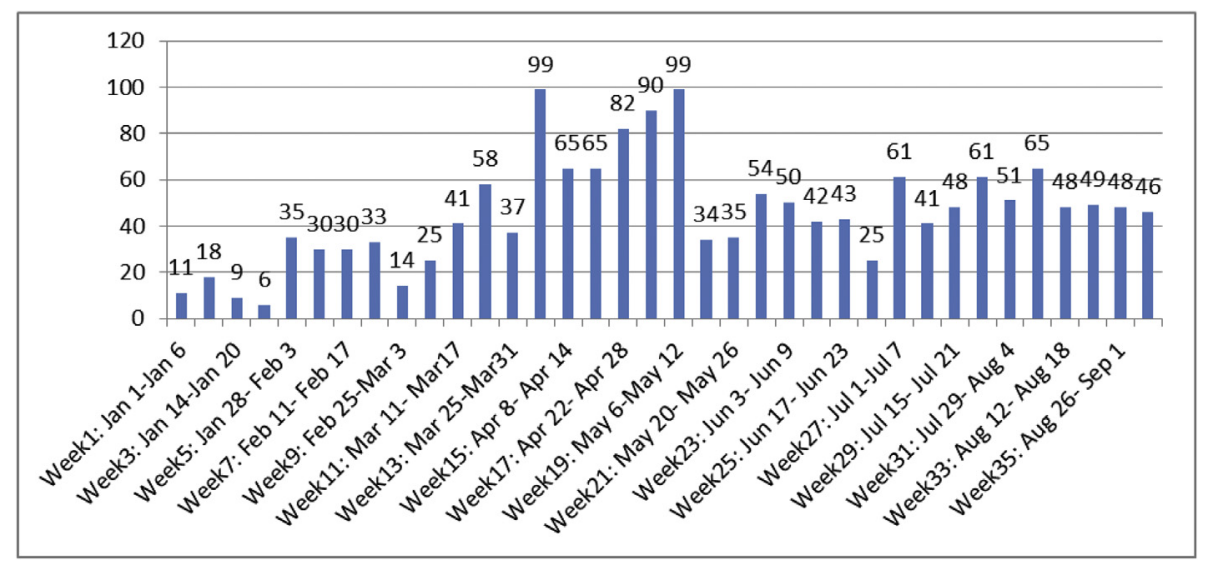

Fig. 2. Suspected mumps cases in Copperbelt province: January 1- September 8, 2015. 


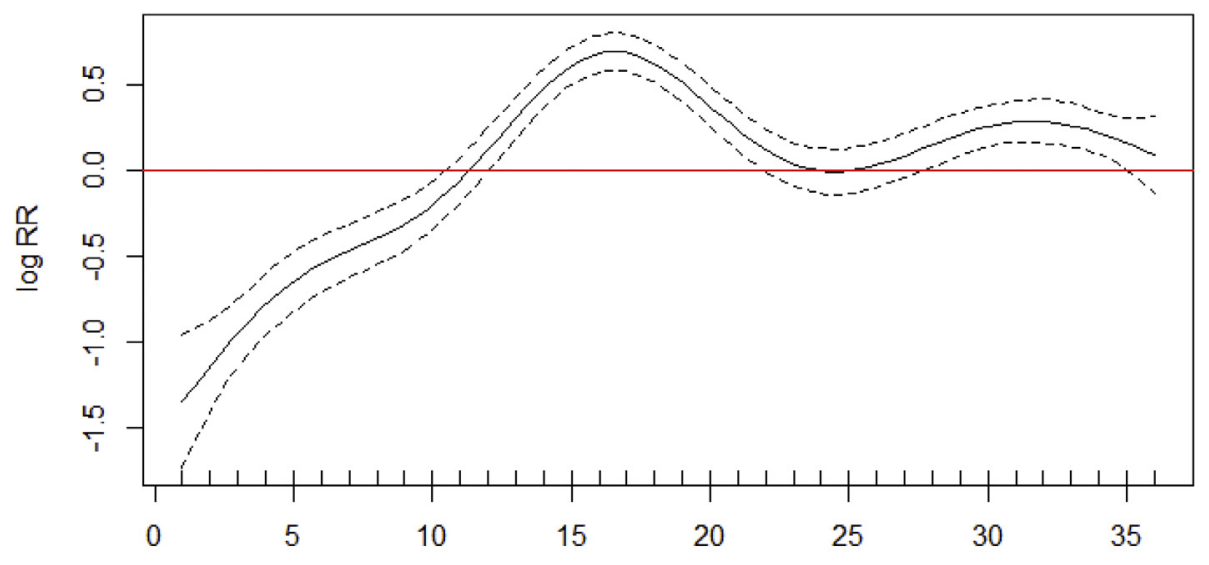

Week: Jan 1-Sep 8, 2015

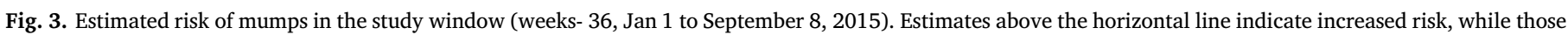
below indicates reduced risk.

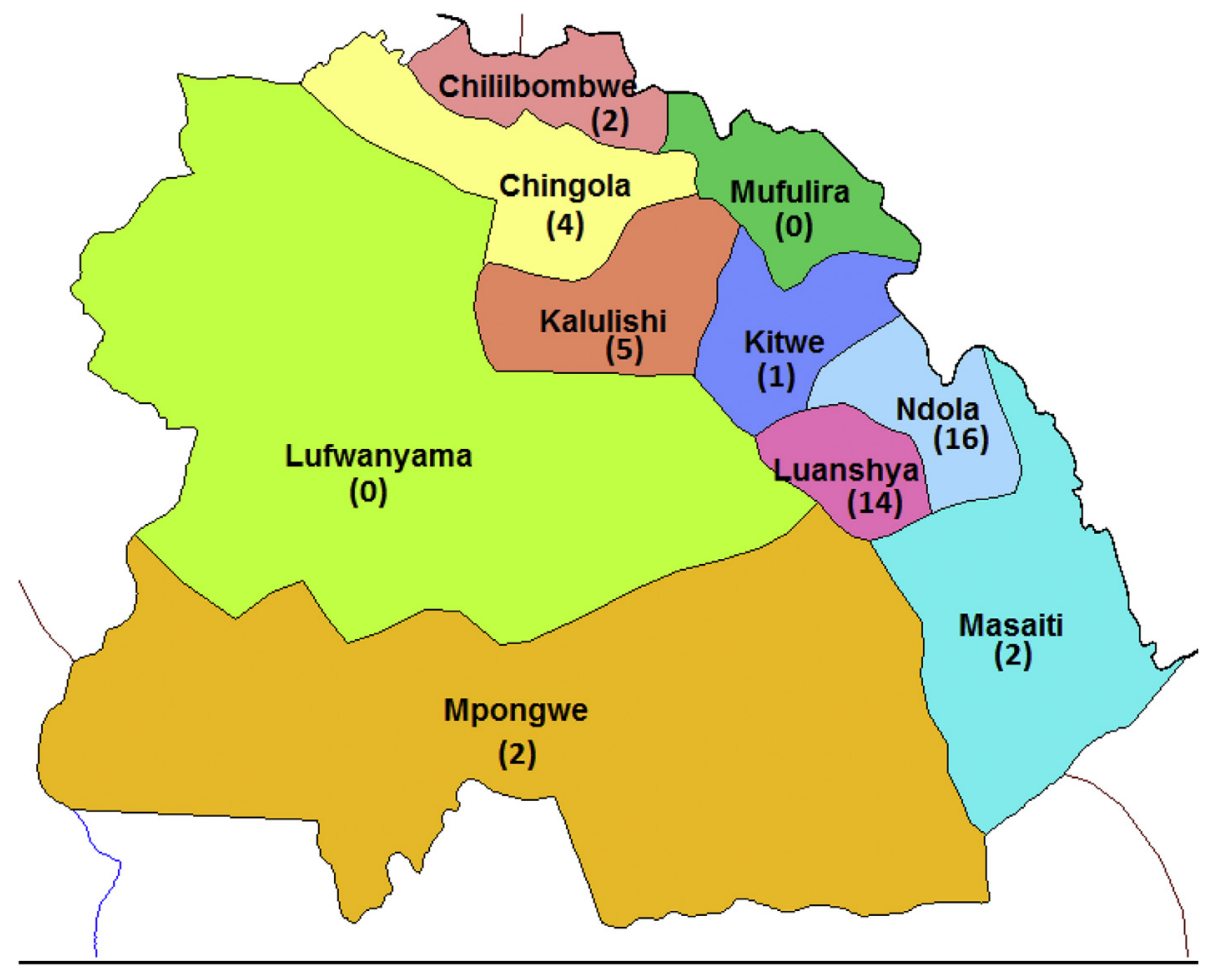

Fig. 4. Spatial distribution of suspected mumps cases in Copperbelt in week 36- Sept 2- Sept. 8, 2015.

disease showed a high incidence of over 175 cases per 100, 000 population, and this is bound to increase since cases are still being seen in most districts on the Copperbelt province. It should be noted that the average monthly number of cases reported in 2015 in the province surpassed the normal national rate of 12 cases per month, between 2011 and 2015. ${ }^{11,12}$

Our results reveal a familiar age pattern, in which children under 10 years are at increased risk, ${ }^{3,4}$ which is hypothesized to relate to the transmission pattern of mumps. First hypothesis advanced in literature is that waning immunity, especially those aged 5-9 years, has contributed to mumps outbreaks in many countries. ${ }^{5,7-9}$ Second, those aged 5-9 years facilitate transmission of mumps in school settings. Close interpersonal contacts in schools prolongs and sustains the transmission of the disease.

The cause of this outbreak is unknown, but possible contacts during the festive season (Christmas and New Year celebrations or the opening of schools in January) may likely have fueled the transmission of the virus in Luanshya district. The fact that the primary case was a child aged 4 years, confirms that this age group is the most vulnerable, especially if these are not immunized of measles or if their immunity has waned. Lack of or waning of immunization may also contribute to the rise in the number of cases of mumps. According to the latest immunization data for Zambia, only 52 districts out of the total 104 districts, have a coverage of above $95 \%$ for first dose of the measles containing vaccine while the rest fall below the stipulated coverage. ${ }^{13}$ The Ministry of Health in Zambia estimates that $6-16 \%$ of the population were susceptible to rubella virus infection.

The importance of this outbreak cannot be over-emphasized. There seem to be a continuous epidemic which started in January 2015, probably with a niche being established in Luanshya. The spatial variation within Luanshya is evident, with strong density in Mikomfwa and Roan township and low density in Fisenge and Mpatamato (Table 1), 
Table 1

Demographic characteristics of mumps cases in Luanshya District, Zambia, January 2015-June 2015 ( $\mathrm{n}=297$ ).

\begin{tabular}{|c|c|c|}
\hline Characteristics & Number (\%) & p-value \\
\hline \multicolumn{3}{|l|}{ Sex } \\
\hline Female & $151(51)$ & \multirow[t]{2}{*}{0.15} \\
\hline Male & $146(49)$ & \\
\hline \multicolumn{3}{|l|}{ Age group } \\
\hline$<4 \mathrm{yr}$ & $115(39)$ & \multirow[t]{5}{*}{$<0.001$} \\
\hline $5-9 \mathrm{yr}$ & $97(33)$ & \\
\hline $10-14 \mathrm{yr}$ & $37(12)$ & \\
\hline $15-20 \mathrm{yr}$ & $18(6)$ & \\
\hline$>20 \mathrm{yr}$ & $30(11)$ & \\
\hline \multicolumn{3}{|l|}{ Residence } \\
\hline Mikomfwa & $130(44)$ & \multirow[t]{5}{*}{$<0.001$} \\
\hline Roan Township & $102(34)$ & \\
\hline Kawama & $32(11)$ & \\
\hline Fisenge & $14(5)$ & \\
\hline Mpatamato & $13(4)$ & \\
\hline \multicolumn{3}{|c|}{ Reporting health centre/clinic: } \\
\hline Mikomfwa & $130(44)$ & \multirow[t]{6}{*}{$<0.001$} \\
\hline Roan Section 9 & $99(33)$ & \\
\hline Kawama & $32(11)$ & \\
\hline Fisenge & $19(6)$ & \\
\hline Mpatamato Section 26 & $14(5)$ & \\
\hline Roan General Hospital & $2(1)$ & \\
\hline \multicolumn{3}{|c|}{ Death and Complications } \\
\hline Death & $0(0)$ & \\
\hline Complications & $0(0)$ & \\
\hline
\end{tabular}

Table 2

Risk factors of mumps in Luanshya district, Copperbelt. Given are the relative risk (RR) and corresponding 95\% confidence interval (CI) based on Poisson regression.

\begin{tabular}{ll}
\hline Variable & $\mathrm{RR}(95 \% \mathrm{CI})$ \\
\hline Sex & \\
Female & 1.00 \\
Male & $0.94(0.73,1.25)$ \\
Age group & \\
$<4$ yr & $3.94(3.02,6.23)$ \\
$5-9$ yr & $3.12(2.11,4.93)$ \\
10-14 yr & $1.21(0.66,2.21)$ \\
15-20 yr & $0.50(0.31,1.03$ \\
$>$ 20 yr & 1.00 \\
Residence & \\
Mikomfwa & $9.27(4.85,15.82)$ \\
Roan Township & $7.25(4.34,13.37)$ \\
Kawama & $2.24(1.21,4.41)$ \\
Fisenge & $1.06(0.46,2.22)$ \\
Mpatamato & 1.00 \\
Reporting health centre/clinic: & \\
Mikomfwa & 1.00 \\
Roan Section 9 & $0.73(0.54,0.99)$ \\
Kawama & $0.21(0.18,0.35)$ \\
Fisenge & $0.17(0.088,0.24)$ \\
Mpatamato Section 26 & $0.14(0.050,0.25)$ \\
Roan General Hospital & $0.012(0.0023,0.058)$ \\
Model fit statistics & \\
Deviance explained & $72.9 \%$ \\
AIC & 331.24 \\
Dispersion statistic & 2.95 \\
\hline
\end{tabular}

however, the variability does not shore any particular parterning with socio-economic distribution, but rather agrees with the reporting health facility. There is a strong possibility that there is a changing epidemiology of the disease in Zambia, and the fact that a different genotype can cause an outbreak, we do not have further information as to the type of genotype associated with the current outbreak. Be as it may, studies have shown that a sera obtained by children within 6 weeks are protective against other strains that may develop. ${ }^{10}$ It therefore remains to conjecture that most of those affected were not immunized with
Measles vaccine, and lack of MMR thereof puts the population at risk. Be as it may, as of September 2016, the Measles Rubella vaccine was introduced.

In conclusion, several factors may contribute to the emergence of mumps outbreaks. Maillet et al. ${ }^{14}$ suggested the following can contribute to new outbreaks: intense exposures, overwhelming the protection offered by the vaccine, combined with non-optimal vaccine coverage, low immunogenicity and waning immunity.

This mumps outbreak considered here has highlighted the difficulties of mumps prevention and control. The delay in confirming the outbreak, which only came in Week 28 , is of concern for an epidemic control, especially if the disease is virulent. We therefore recommend that, first, continued surveillance be maintained so that cases are identified, contacts are traced, and inform implementation of appropriate infection control measures, through grounded outbreak management. Second, continued Information, Education and Communication (IEC) on good hygiene practices, modes of transmission and risk factors in schools and other public places is recommended. Third, there is need to establish local emergency health services linking schools and health facilities. Furthermore, health authorities should make concerted efforts to enable diagnostic tests for mumps within the country to permit confirmation of more cases, while at the same time we advocate for increased coverage of Measles RubellaVaccine in Zambia, through yearly mass vaccination campaign as done in previous years, such as in 2010, 2012 and 2016, particularly targeting those with low immunization coverage. At the same time, the results should advocate for the introduction of Measles, Mumps \& Rubella (MMR) vaccine.

\section{Conflicts of interest}

We declare there is no conflict of interest.

\section{Funding}

No funding from any organization.

\section{Competing interests}

None.

\section{Ethical approval}

None.

\section{Acknowledgement}

We acknowledge support given by the Provincial Medical Team in Copperbelt, Zambia.

\section{References}

1. Hviid A, Rubin S, Mühlemann K. Mumps. Lancet. 2008;371:932-944.

2. Simpson REH. Infectiousness of communicable disease in the household: measles, chickenpox and mumps). Lancet. 1952;260:549-554.

3. Kontio M, Jokinen S, Paunio M, Peltola H, Davidkin I. Waning antibody levels and avidity: implications for MMR vaccine-induced protection. J Infect Dis. 2012;206:1542-1548.

4. Anis E, Grotto I, Moerman L, Warshavsky B, Slater PE, Lev B. Mumps outbreak in Israel's highly vaccinated society: are two doses enough? Epidemiol Infect. 2012;140:439-446.

5. Braeye T, Linina I, De Roy R, et al. Mumps increase in Flanders, Belgium, 2012-2013: results from temporary mandatory notification and a cohort study among university students. Vaccine. 2014;32:4393-4398.

6. R Core Team. R: A Language and Environment for Statistical Computing. Vienna, Austria: R Foundation for Statistical Computing; 2018https://www.R-project.org/.

7. Marin M, Quinlisk P, Shimabukuro T, Sawhney C, Brown C, Lebaron CW. Mumps vaccination coverage and vaccine effectiveness in a large outbreak among college students-Iowa, 2006. Vaccine. 2008;26:3601-3607.

8. Cortese MM, Jordan HT, Curns AT, et al. Mumps vaccine performance among 
university students during a mumps outbreak. Clin Infect Dis. 2008;46:1172-1180.

9. Park SH. Resurgence of mumps in Korea. Infect Chemother. 2015;47(1):1-11.

10. Rubin SA, Link MA, Sauder CJ, Ngo L, Rima BK, Duprex P. Recent mumps outbreaks in vaccinated populations: no evidence of immune escape. J Virol. 2012;86:615-620.

11. WHO. Monitoring and surveillance - mumps. Available at: http://www.who.int/ immunization/monitoring_surveillance/burden/vpd/surveillance_type/passive/ mumps/en/ Accessed on 27th May, 2018.

12. Berger S. Mumps: Global Status. Gideon Informatics Inc; 2018.

13. Central Statistical Office (CSO), Ministry of Health (MOH), and ICF International. 2015 Zambia Demographic and Health Survey Report. March 2017; March 2017.

14. Maillet M, Bouvat E, Robert N, et al. Mumps outbreak and laboratory diagnosis. $J$ Clin Virol. 2015 Jan;62:14-19. https://doi.org/10.1016/j.jcv.2014.11.004. 